\title{
Crizotinib versus alectinib for treatment of ALK-positive non-small cell lung cancer: a pooled analysis of the ALEX, ALESIA and J- ALEX clinical trials
}

\section{Qinghua Zeng}

The First Affiliated Hospital of Nanchang University

Xiquan Zhang

Jiangxi provincial People's Hospital Affiliated to Nanchang University

Shan He

The First Affiliated Hospital of Nanchang University

\section{Zhiyong Zhou}

Jiangxi provincial People's Hospital Affiliated to Nanchang University

Luping Xia

The First Affiliated Hospital of Nanchang University

\section{Wenxiong Zhang}

The Second Affiliated Hospital of Nanchang University

Lin Zeng ( $\nabla$ figozl@163.com )

Jiangxi provincial People's Hospital Affiliated to Nanchang University https://orcid.org/0000-0003-2537-2938

\section{Research}

Keywords: Crizotinib, Alectinib, anaplastic lymphoma kinase, Non-small cell lung cancer, Meta-analysis.

Posted Date: September 16th, 2020

DOI: https://doi.org/10.21203/rs.3.rs-75474/v1

License: @ (i) This work is licensed under a Creative Commons Attribution 4.0 International License. Read Full License 


\section{Abstract}

Background: Crizotinib and alectinib were the two most commonly used anaplastic lymphoma kinase (ALK) inhibitors for ALK-positive non-small cell lung cancer (NSCLC). We compared their antitumor efficacies and adverse effects based on a pooled analysis of the ALEX, ALESIA and J-ALEX clinical trials.

Methods: Seven databases were searched for eligible articles. The primary endpoints included overall survival (OS), progression-free survival (PFS), central nervous system (CNS)-PFS, drug responses and adverse effects (AEs).

Results: Three randomized controlled clinical trials (ALEX, ALESIA and J-ALEX) with a total of 7 articles and 697 patients were included. Compared with crizotinib, alectinib exhibited superior efficacy in PFS (HR [hazard ratio]: 0.35, [0.25-0.49], $p<0.00001$ ), OS (HR: 0.66, [0.47-0.92], $p=0.02$ ), CNS-PFS (HR: 0.17, [0.11-0.24], $p<0.00001)$, duration of response (HR: 0.31, [0.23-0.42], $p<0.00001$ ), objective response rate (ORR) (Risk ratio [RR]: 0.87, [0.80-0.94], $p=0.0003$ ), partial response (PR) (RR: 0.88, [0.81-0.96], $p=0.004$ ), and grade 3-5 AEs (RR: 1.43, [1.09-1.87], $p=0.009$ ). Additionally, the survival advantages of alectinib compared with crizotinib increased with alectinib's prolongation of survival time. The disease control rate, complete response and total AEs were comparable between the two groups. A greater increase in constipation, nausea, diarrhea, alanine aminotransferase, vomiting, aspartate aminotransferase, peripheral edema, dysgeusia, and visual impairment as well as a greater decrease in appetite and neutrophil count were associated with the crizotinib group

Conclusions: In both antitumor efficacy and safety, alectinib appears to be superior to crizotinib for the treatment of ALK-positive NSCLC.

\section{Introduction}

Over the last decade, lung cancer has become the leading cause of cancer-related death, and $80 \%$ of all lung cancers are non-small cell lung cancer (NSCLC) ${ }^{[1]}$. Approximately 3\%-7\% of all patients with NSCLC have anaplastic lymphoma kinase (ALK)-positive disease ${ }^{[2]}$. Crizotinib, the first clinically established ALK inhibitor, exhibits satisfactory antitumor efficacy and safty compared with chemotherapy for treatment of ALK-positive NSCLC ${ }^{[3,4]}$. However, frequent crizotinib resistance and poor central nervous system (CNS) efficacy are very troubling for clinicians ${ }^{[5]}$.

Alectinib, the most commonly used second-generation ALK inhibitor, is effective against several ALK-mutations and exhibits high selectivity and CNS activity ${ }^{[6,7]}$. In the AF-001JP clinical trial, Seto et al. ${ }^{[8]}$ demonstrated that alectinib is highly effective for the treatment of ALK-positive NSCLC. However, whether alectinib can replace crizotinib as the first-line treatment for patients with ALKpositive NSCLC remains controversial ${ }^{[9,10]}$. Peters et al. ${ }^{[11]}$ reported that alectinib achieves better progression-free survival (PFS) and has less adverse effects (AEs) than crizotinib for treatment of ALK-positive NSCLC. Hida et al. ${ }^{[12]}$ and Nishio et al. ${ }^{[13]}$ suggested that alectinib could prevent new brain metastasis and avert the progression of brain metastases. In the ALESIA clinical trial, Zhou et al. ${ }^{[14]}$ confirmed that alectinib treatment results in better clinical benefits, e.g., progression-free survival (PFS) and CNS-PFS, in ALK-positive NSCLC. However, significant advantage of overall survival (OS) with statistical significance was still not comfirmed in the ALEX,

ALESIA and J-ALEX trials ${ }^{[11,12,14]}$. Bedas et al. ${ }^{[15]}$ reported that crizotinib and alectinib treatments are associated with similar PFS and OS in elderly patients with advanced ALK-positive NSCLC.

To further clarify this debate, we compared the efficacy and safety of crizotinib versus alectinib for treatment of ALK-positive NSCLC by performing a meta-analysis of the relevant literature.

\section{Materials And Methods}

We conducted this study according to the Preferred Reporting Items for Systematic Reviews and Meta-Analysis guidelines (PRISMA) (Table S1).

\section{Search strategy}

PubMed, EMBASE, Scopus, Ovid MEDLINE, Web of Science, Cochrane Library, ScienceDirect and Google Scholar were rigorously searched for eligible randomized, controlled clinical trials (RCTs) from inception to May 5, 2020. As key words, we used "lung cancer", 
"crizotinib" and "alectinib". We also searched the reference lists of the included RCTs to identify additional eligible studies. Details can be found in Table $\mathbf{S 2}$.

\section{Selection criteria}

Studies which obeyed these criteria would be enrolled in accordance with PICOS (Participants, Intervention, Control, Outcome, Study design):

(1) Population (P): patients with ALK-positive NSCLC.

(2) Intervention (I) and comparison (C): crizotinib vs. alectinib.

(3) Outcomes (0): anti-tumor efficacy and AEs (see the data extraction).

(4) Study design (S): RCTs published in English.

The following articles were excluded: articles without initial data, meta-analyses, conference articles, case reports, and articles from the same experimental center on the same topic. Different articles that focused on the same trial were included if they contained different outcomes; however, when analyzing the same outcome, only the updated data were used.

\section{Data extraction}

The following data were extracted by two independent investigators: the study characteristics (e.g., publication date, first author, and design), participants' characteristics (e.g., quantity, sex, and age), cancer characteristics (e.g., histopathology, stage, and ALK status), anti-tumor efficacy (e.g., OS, PFS, CNS-PFS, and drug responses), and number of AEs (total AEs, grade 3-5 AEs, treatment discontinuation, dose reduction, and dose interruption). All disagreements between the two investigators were resolved through reexamination and discussion.

\section{Outcome Assessments}

OS, PFS and CNS-PFS were the primary endpoints analyzed. In addition to analyzing the time-to-event data, we also compared the rates of survival (overall survival rate [OSR], progression-free survival rate [PFSR] and central nervous system progression-free survival rate [CNS-PFSR]) at 6, 12, 18, 24 and 30 months (OSR 6-30 m, PFSR 6-30 m and CNS-PFSR 6-30 m) between the two groups. Additionally, we analyzed the PFS according to the following subgroups: age, sex, smoking status, CNS metastases at baseline, race category, treatment line, previous brain radiation, disease stage and the ALK testing method.

\section{Quality assessment}

The quality of RCTs was assessed using the 5-point Jadad scale and the Cochrane Risk Assessment Tool. The Jadad scale primarily evaluates quality based on the following three factors: randomization, blinding, and patient inclusion. A study is regarded as high quality if it receives a score of $\geq 3$ points ${ }^{[16]}$. The Cochrane Risk Assessment Tool primarily focuses on the bias of selection, performance, detection, attrition, and reporting, and the risk is assessed as low, unclear or high risk ${ }^{[17]}$. Then, the results are presented as a risk of bias graph.

The quality of the results was assessed using the Grading of Recommendations, Assessment, Development, and Evaluation (GRADE) method ${ }^{[18]}$. The GRADE approach primarily focuses on bias, discordance, indirectness, inaccuracy and publication bias. The results include four levels: very low, low, medium and high.

\section{Statistical analysis}

We used Review Manager 5.3 software (Nordic Cochrane Center, Oxford, UK) to evaluate the pooled data. The hazard ratio (HR) was used to analyze the survival data (OS, PFS and CNS-PFS). All of the HR data were directly extracted from the included studies. When the $H R<1$, then the results supported the alectinib group. The risk ratio (RR) was used to analyze the dichotomous variables (drug responses, OSR, PFSR, CNS-PFSR and AEs). When the RR $>1$, then the results supported the alectinib group, as in the analysis of AEs,

or the results supported the crizotinib group, as in the analysis of OSR, PFSR, CNS-PFSR and AEs. We used the $P^{2}$ statistic and $\chi^{2}$ test to evaluate the heterogeneity. If $R^{2}<50 \%$ or $p>0.1$, which indicates no significant heterogeneity, then we used a fixed-effects model; otherwise, we used a random-effects model. Statistical significance was indicated when $P<0.05$. Publication bias was assessed by performing a visual check of the funnel plots. 


\section{Results}

\section{Search results}

Three randomized, controlled clinical trials (ALEX, ALESIA and J-ALEX) with a total of 7 articles and 697 patients (317 patients in the crizotinib arm and 380 patients in the alectinib arm) were included for the final analysis ${ }^{[11-14,22-24]}$ (Fig. 1). The ALESIA and J-ALEX trials ${ }^{[12,14]}$ were conducted in Asia, but the ALEX trial included participants from 32 countries all over the world ${ }^{[11]}$. All three of these studies are high quality according to the Cochrane Risk of Bias Tool (Figure S1) and the Jadad scale (Table S3). According to the GRADE method, all of the results were of medium-high quality (Table S4). The essential information from the ALEX, ALESIA and JALEX clinical trials is summarized in Table 1. 
Table 1

Characteristics of the three randomized controlled trials (ALEX, ALESIA and J-ALEX).

\begin{tabular}{|c|c|c|c|c|c|c|}
\hline Study & \multicolumn{2}{|l|}{ ALESIA } & \multicolumn{2}{|l|}{ ALEX } & \multicolumn{2}{|l|}{ J-ALEX } \\
\hline Register number & \multicolumn{2}{|c|}{ NCT02838420 } & \multicolumn{2}{|c|}{ NCT02075840 } & \multicolumn{2}{|c|}{ JapicCTI-132316 } \\
\hline Design & \multicolumn{2}{|l|}{$\mathrm{RCT}$} & \multicolumn{2}{|l|}{$\mathrm{RCT}$} & \multicolumn{2}{|l|}{$\mathrm{RCT}$} \\
\hline Clinical trial stage & \multicolumn{2}{|l|}{ Phase III } & \multicolumn{2}{|l|}{ Phase III } & \multicolumn{2}{|l|}{ Phase III } \\
\hline Treatment line & \multicolumn{2}{|l|}{1} & \multicolumn{2}{|l|}{1} & \multicolumn{2}{|l|}{1 or 2} \\
\hline Inculded articles & \multicolumn{2}{|c|}{ Zhou 2019[14] } & \multicolumn{2}{|c|}{$\begin{array}{l}\text { Peters 2017[11], Gadgeel 2018[22], } \\
\text { Camidge 2019[23] }\end{array}$} & \multicolumn{2}{|c|}{$\begin{array}{l}\text { Hida 2017[12], Nishio 2018[13], } \\
\text { Nakagawa 2020[24] }\end{array}$} \\
\hline Country & \multicolumn{2}{|c|}{$\begin{array}{l}\text { China, South Korea and } \\
\text { Thailand }\end{array}$} & \multicolumn{2}{|c|}{ Multi countries } & \multicolumn{2}{|l|}{ Japen } \\
\hline Period & \multicolumn{2}{|c|}{ 2016.08-2017.05 } & \multicolumn{2}{|c|}{ 2014.08-2016.01 } & \multicolumn{2}{|c|}{ 2013.11-2015.08 } \\
\hline Treatment arms & Crizotinib & Alectinib & Crizotinib & Alectinib & Crizotinib & Alectinib \\
\hline Dose & $\begin{array}{l}250 \mathrm{mg} \\
\text { bid, Oral }\end{array}$ & $\begin{array}{l}600 \mathrm{mg} \\
\text { bid, Oral }\end{array}$ & $\begin{array}{l}250 \mathrm{mg} \text { bid, } \\
\text { Oral }\end{array}$ & 600 mg bid, Oral & $\begin{array}{l}250 \mathrm{mg} \mathrm{bid} \text {, } \\
\text { Oral }\end{array}$ & $300 \mathrm{mg}$ bid, Oral \\
\hline Patients (n) & 62 & 125 & 151 & 152 & 104 & 103 \\
\hline $\operatorname{Sex}(M / F)$ & $34 / 28$ & $61 / 61$ & $64 / 87$ & $68 / 84$ & $41 / 63$ & $41 / 62$ \\
\hline Median age (year) & 49 & 51 & 54 & 58 & 59.5 & 61 \\
\hline \multicolumn{7}{|l|}{ Race } \\
\hline Asian & 62 & 125 & 69 & 69 & 104 & 103 \\
\hline Non-Asian & 0 & 0 & 82 & 83 & 0 & 0 \\
\hline $\begin{array}{l}\text { Median duration of } \\
\text { therapy (months) }\end{array}$ & 12.6 & 14.7 & 10.7 & 17.9 & - & - \\
\hline ECOG status & $0-2$ & & $0-2$ & & $0-2$ & \\
\hline $\begin{array}{l}\text { Follow-up } \\
\text { duration (months) }\end{array}$ & 15 & 16.2 & 22.8 & 27.8 & 42.2 & 42.4 \\
\hline Pathology & \multicolumn{2}{|c|}{ ALK positive NSCLC } & \multicolumn{2}{|c|}{ ALK positive NSCLC } & \multicolumn{2}{|c|}{ ALK positive NSCLC } \\
\hline ALK test method & \multicolumn{2}{|l|}{$\mathrm{IHC}$} & \multicolumn{2}{|l|}{$\mathrm{IHC}$} & \multicolumn{2}{|c|}{$\begin{array}{l}190 \text { patients by IHC and FISH, } 17 \\
\text { patients by RT-PCR }\end{array}$} \\
\hline Stage & \multicolumn{2}{|l|}{ IIIb, IV } & IIIb, IV & & III, IV & \\
\hline Brain metastasis & & & & & & \\
\hline Measurable & 7 & 17 & 22 & 21 & 29 & 15 \\
\hline Non-measurable & 16 & 27 & 36 & 43 & & \\
\hline No & 39 & 81 & 93 & 88 & 75 & 89 \\
\hline $\begin{array}{l}\text { Tumor response } \\
\text { assessment }\end{array}$ & RECIST, ve & on 1.1 & RECIST, vers & & RECIST, vers & \\
\hline
\end{tabular}

Abbreviations: RCT: randomized controlled trial; NSCLC: non-small cell lung cancer; Bid: twice a day; ECOG: Eastern Cooperative Oncology Group; M/F: male/female; ALK: anaplastic lymphoma kinase; NCI-CTCAE, National Cancer Institute Common Terminology Criteria for Adverse; IHC: immunohistochemistry; FISH: fluorescence in situ hybridization; MRI: magnetic resonance imaging; RECIST: Response Evaluation Criteria In Solid Tumors; RT-PCR: Reverse Transcription-Polymerase Chain Reaction.

a Multi countries inculding Australia, Bosnia and Herzegovina, Brazil, Canada, Chile, China, Costa Rica, Denmark, Dominican Republic, Egypt, France, Germany, Greece, Guatemala, Hong Kong, Israel, Italy, Mexico, New Zealand, Peru, Poland, Portugal, Republic of Korea, Russia, Serbia, Singapore, Spain, Switzerland, Taiwan, Thailand, Turkey, Ukraine, United Kingdom and USA. 


\begin{tabular}{|c|c|c|c|}
\hline Study & ALESIA & ALEX & J-ALEX \\
\hline $\begin{array}{l}\text { Brain metastasis } \\
\text { assessment }\end{array}$ & MRI in all patients & MRI in all patients & MRI in all patients \\
\hline $\begin{array}{l}\text { Adverse events } \\
\text { assessment }\end{array}$ & NCl-CTCAE, version 4.0 & NCl-CTCAE, version 4.0 & NCI-CTCAE, version 4.0 \\
\hline \multirow{3}{*}{$\begin{array}{l}\text { Frequency of disease } \\
\text { assessment }\end{array}$} & \multirow[t]{3}{*}{ Every 8 weeks until DP } & \multirow[t]{3}{*}{ Every 8 weeks until DP } & Every 4 weeks until week 12 \\
\hline & & & $\begin{array}{l}\text { Every } 8 \text { weeks from week } 12 \text { to } \\
\text { week } 76\end{array}$ \\
\hline & & & every 12 weeks thereafter until DP \\
\hline Funding & $\begin{array}{l}\text { F. Hoffmann-La Roche, } \\
\text { Ltd. }\end{array}$ & F. Hoffmann-La Roche, Ltd. & Chugai Pharmaceutical Co, Ltd. \\
\hline \multicolumn{4}{|c|}{$\begin{array}{l}\text { Abbreviations: RCT: randomized controlled trial; NSCLC: non-small cell lung cancer; Bid: twice a day; ECOG: Eastern Cooperative } \\
\text { Oncology Group; M/F: male/female; ALK: anaplastic lymphoma kinase; NCI-CTCAE, National Cancer Institute Common } \\
\text { Terminology Criteria for Adverse; IHC: immunohistochemistry; FISH: fluorescence in situ hybridization; MRI: magnetic resonance } \\
\text { imaging; RECIST: Response Evaluation Criteria In Solid Tumors; RT-PCR: Reverse Transcription-Polymerase Chain Reaction. }\end{array}$} \\
\hline
\end{tabular}

\section{Anti-tumor efficacy}

Three studies compared the OS of the alectinib group versus the crizotinib group (heterogeneity: $P=55 \%, P=0.02$ ). The OS of the alectinib group was better than that of the crizotinib group (HR: 0.66, 95\% confidence interval [Cl]: [0.47-0.92], $p=0.02$; Fig. 2). The OSR at all time points tended to favor the alectinib group but was not statistically significant (OSR-6 m, RR: 0.97, [0.91-1.04], $p=0.36$; OSR-12 m, RR: 0.89, [0.79-1.02], $p=0.09$; OSR-18 m, RR: 0.83, [0.68-1.00], $p=0.05$; OSR-24 m, RR: 0.74, [0.50-1.09], $p=0.13$; and OSR$30 \mathrm{~m}$, RR: 0.76 , [0.52-1.11], $p=0.16$; Figure S2). With prolonged survival, the OS advantage of alectinib increased compared with that of crizotinib (Fig. 3A and Figure S3A).

Three studies compared the PFS of the alectinib group versus the crizotinib group (heterogeneity: $R=56 \%, P=0.10$ ). The PFS of the alectinib group was better than that of the crizotinib group (HR: 0.35, [0.25-0.49], $p<0.00001$; Fig. 2). The PFSR at all time points significantly favored the alectinib group (PFSR-6 m, RR: 0.87, [0.81-0.95], $p=0.0009$; PFSR-12 m, RR: 0.63, [0.55-0.72], $p<0.00001$; PFSR-18 m, RR: 0.51, [0.43-0.62], $p<0.00001$; PFSR-24 m, RR: 0.38, [0.27-0.53], $p<0.00001$; and PFSR-30 m, RR: 0.39, [0.27-0.56], $p$ $<0.00001$; Figure S4). With prolonged survival, the PFS advantage of alectinib increased compared with that of crizotinib (Fig. 3B and Figure S3B). In the subgroup analysis, significant changes were not observed in PFS according to age, sex, race category, or treatment line. The following might be unfavorable factors for alectinib treatment: smoking status = active smoker, Eastern Cooperative Oncology Group-performance status [ECOG-PS] $=2$, disease stage = postoperative recurrence, and the ALK testing method = reverse transcriptionpolymerase chain reaction (RT-PCR). However, baseline CNS metastases and previous brain radiation might be favorable factors for alectinib treatment (Fig. 4).

Three studies compared the CNS-PFS of the alectinib group versus the crizotinib group (heterogeneity: $P=0 \%, P=0.71$ ). The CNS-PFS of the alectinib group was better than the crizotinib group (HR: 0.17, [0.11-0.24], $p<0.00001$; Fig. 2). The CNS-PFSR at all time points significantly favored the alectinib group (CNS-PFSR-6 m, RR: 0.88, [0.80-0.96], $p=0.005$; CNS-PFSR-12 m, RR: 0.70, [0.64-0.76], $p<$ 0.00001; CNS-PFSR-18 m, RR: 0.66, [0.60-0.73], $p<0.00001$; CNS-PFSR-24 m, RR: 0.58, [0.51-0.66], $p<0.00001$; and CNS-PFSR-30 m, RR: 0.58, [0.47-0.72], $p<0.00001$; Figure S5). With prolonged survival, the CNS-PFS advantage of alectinib increased compared with that of crizotinib (Fig. 3C and Figure S3C).

Three studies compared the DOR of the alectinib group versus the crizotinib group (heterogeneity: $P=0 \%, P=0.41$ ). The DOR of the alectinib group was better than the crizotinib group (HR: 0.31, [0.23-0.42], $p<0.00001$; Fig. 2). The objective response rate [ORR] (74.45\% vs. $86.58 \%$, RR: $0.87,[0.80-0.94], p=0.0003)$ and the partial response [PR] (71.92\% vs. $82.11 \%, \mathrm{RR}: 0.88,[0.81-0.96], p=$ $0.004)$ were better in the alectinib group. The disease control rate [DCR] (90.54\% vs. $94.21 \%, \mathrm{RR}: 0.95,[0.88-1.04], p=0.27)$ and the 
complete response [CR] (2.52\% vs. 4.47\%, RR: 0.57 , [0.26-1.32], $p=0.20)$ were similar between the groups. Due to the high ORR and similar DCR in the alectinib group, less stable disease [SD] (16.09\% vs. 7.63\%, RR: 2.01, [13.1-3.10], $p=0.001)$ was found in the alectinib group (Fig. 5).

Subgroup analysis was conducted according to patients have baseline CNS metastases or not. In patients with CNS lesions at baseline, alectinib group was better than the crizotinib group in PFS (HR: 0.23, [0.11-0.49], $p=0.0001$ ), systemic progression without previous CNS disease progression (HR: 0.35, [0.15-0.83], $p=0.02$ ) and CNS progression without previous systemic disease progression(HR: 0.18, [0.09-0.36], $p<0.00001)$. In patients without CNS lesions at baseline, alectinib group was also better than the crizotinib group in PFS (HR: 0.43, [0.30-0.60], $p<0.00001)$ and CNS progression without previous systemic disease progression (HR: 0.14 , [0.06-0.33], $p<0.00001)$. Similar death without previous CNS or systemic disease progression was found bwtween the two groups in patients with or without CNS lesions at baseline (Table S5).

According to the assessment of independent review committee (IRC), alectinib group was better than the crizotinib group in OS (HR: 0.66, [0.47-0.92], $p=0.02$ ), PFS (HR: 0.42, [0.34-0.52], $p<0.00001)$, CNS-PFS (HR: 0.18, [0.11-0.27], $p<0.00001$ ) and DOR (HR: 0.32, [0.17-0.60], $p=0.0004)$. According to the assessment of investigators, alectinib group was also better than the crizotinib group in OS (HR: 0.66, [0.47-0.92], $p=0.02$ ), PFS (HR: 0.35, [0.25-0.49], $p<0.00001)$, CNS-PFS (HR: 0.14, [0.06-0.31], $p<0.00001$ ) and DOR (HR: 0.31 , [0.22-0.43], $p<0.00001$ ) (Table S6). Table $\mathbf{S 6}$ also showed the results of responses and CNS responses assessed by IRC and investigators.

\section{Toxicity}

In summary, crizotinib treatment was associated with more grade $3-5$ AEs (53.63\% vs. $37.37 \%$, RR: 1.43 , [1.09-1.87], $p=0.009)$. The total AEs ( $98.74 \%$ vs. $98.16 \%$, RR: 1.01 , [0.99-1.03], $p=0.40$ ), serious AEs (29.02\% vs. $24.47 \%$, RR: $1.12,[0.88-1.44], p=0.34)$, fatal AEs (3.15\% vs. $2.11 \%$, RR:1.51, [0.62-3.69], $p=0.37$ ), AEs leading to treatment discontinuation (15.77\% vs. 10.79\%, RR: 1.37, [0.93-2.02], $p$ $=0.11)$, AEs leading to dose reduction (21.13\% vs. $19.86 \%$, RR: $1.11,[0.77-1.60], p=0.57)$, AEs leading to dose interruption (39.43\% vs. $26.58 \%$, RR: $1.38,[0.90-2.12], p=0.15)$, and death ( $0 \%$ vs. $0.53 \%, \mathrm{RR}: 0.20,[0.01-4.16], p=0.30)$ were comparable between the two groups (Table 2).

Table 2

Summary of adverse events.

\begin{tabular}{|c|c|c|c|c|c|c|c|c|c|}
\hline \multirow[t]{2}{*}{ Adverse events } & \multirow{2}{*}{$\begin{array}{l}\text { Studies } \\
\text { involved }\end{array}$} & \multicolumn{2}{|l|}{ Crizotinib } & \multicolumn{2}{|l|}{ Alectinib } & \multirow{2}{*}{$\begin{array}{l}\text { Risk } \\
\text { ratio }\end{array}$} & \multirow{2}{*}{$\begin{array}{l}95 \% \\
\mathrm{Cl}\end{array}$} & \multirow[t]{2}{*}{$R(\%)$} & \multirow[t]{2}{*}{$\mathbf{P}$} \\
\hline & & Event/total & $\%$ & Event/total & $\%$ & & & & \\
\hline Total adverse events & 3 & $313 / 317$ & $98.74 \%$ & $373 / 380$ & $98.16 \%$ & 1.01 & $\begin{array}{l}0.99- \\
1.03\end{array}$ & 0 & 0.4 \\
\hline Grade 3-5 adverse events & 3 & $170 / 317$ & $53.63 \%$ & $142 / 380$ & $37.37 \%$ & 1.43 & $\begin{array}{l}1.09- \\
1.87\end{array}$ & 59 & 0.009 \\
\hline Serious adverse events & 3 & $92 / 317$ & $29.02 \%$ & $93 / 380$ & $24.47 \%$ & 1.12 & $\begin{array}{l}0.88- \\
1.44\end{array}$ & 14 & 0.34 \\
\hline Fatal adverse events & 2 & $10 / 213$ & $4.69 \%$ & $8 / 277$ & $2.89 \%$ & 1.51 & $\begin{array}{l}0.62- \\
3.69\end{array}$ & 0 & 0.37 \\
\hline $\begin{array}{l}\text { Adverse event leading to } \\
\text { treatment discontinuation }\end{array}$ & 3 & $50 / 317$ & $15.77 \%$ & $41 / 380$ & $10.79 \%$ & 1.37 & $\begin{array}{l}0.93- \\
2.02\end{array}$ & 16 & 0.11 \\
\hline $\begin{array}{l}\text { Adverse event leading to dose } \\
\text { reduction }\end{array}$ & 2 & $45 / 213$ & $21.13 \%$ & $55 / 277$ & $19.86 \%$ & 1.11 & $\begin{array}{l}0.77- \\
1.60\end{array}$ & 0 & 0.57 \\
\hline $\begin{array}{l}\text { Adverse event leading to dose } \\
\text { interruption }\end{array}$ & 3 & $125 / 317$ & $39.43 \%$ & $101 / 380$ & $26.58 \%$ & 1.38 & $\begin{array}{l}0.90- \\
2.12\end{array}$ & 72 & 0.15 \\
\hline Death & 2 & $0 / 317$ & $0.00 \%$ & $2 / 380$ & $0.53 \%$ & 0.2 & $\begin{array}{l}0.01- \\
4.16\end{array}$ & - & 0.3 \\
\hline
\end{tabular}

Subgroup analysis of the total AEs found that there was a greater increase in constipation, nausea, diarrhea, alanine aminotransferase (ALT), vomiting, aspartate aminotransferase (AST), peripheral edema, dysgeusia, and visual impairment as well as a greater decrease 
in appetite and neutrophil count associated with the crizotinib group. However, increased blood bilirubin, bronchitis and anemia were found in the alectinib group. Total AEs with an incidence greater than $10 \%$ according to the combination of the crizotinib group and the alectinib group are summarized in Table 3, Table S7 and Table S8. 
Table 3

Total adverse events with an incidence of greater than $10 \%$ according to combination of the two groups.

\begin{tabular}{|c|c|c|c|c|c|c|c|c|c|c|}
\hline \multirow[t]{2}{*}{ Adverse events } & \multirow{2}{*}{$\begin{array}{l}\text { Studies } \\
\text { involved }\end{array}$} & \multicolumn{2}{|l|}{ Crizotinib } & \multicolumn{2}{|l|}{ Alectinib } & \multirow{2}{*}{$\begin{array}{l}\text { Total } \\
\text { incidence }\end{array}$} & \multirow{2}{*}{$\begin{array}{l}\text { Risk } \\
\text { ratio }\end{array}$} & \multirow[t]{2}{*}{$95 \% \mathrm{Cl}$} & \multirow[t]{2}{*}{$R(\%)$} & \multirow[t]{2}{*}{$\mathbf{P}$} \\
\hline & & Event/total & $\%$ & Event/total & $\%$ & & & & & \\
\hline Constipation & 2 & $79 / 166$ & $47.59 \%$ & $84 / 228$ & $36.84 \%$ & $41.37 \%$ & 1.29 & $\begin{array}{l}1.02- \\
1.63\end{array}$ & 0 & 0.03 \\
\hline Nausea & 3 & $177 / 317$ & $55.84 \%$ & $47 / 380$ & $12.37 \%$ & $32.14 \%$ & 4.15 & $\begin{array}{l}3.13- \\
5.51\end{array}$ & 40 & $\begin{array}{l}< \\
0.00001\end{array}$ \\
\hline Diarrhea & 3 & $177 / 317$ & $55.84 \%$ & $45 / 380$ & $11.84 \%$ & $31.85 \%$ & 4.67 & $\begin{array}{l}2.84- \\
7.68\end{array}$ & 62 & $\begin{array}{l}< \\
0.00001\end{array}$ \\
\hline ALT increased & 3 & $115 / 317$ & $36.28 \%$ & $89 / 380$ & $23.42 \%$ & $29.27 \%$ & 1.83 & $\begin{array}{l}1.17- \\
2.86\end{array}$ & 68 & 0.008 \\
\hline Vomiting & 3 & $146 / 317$ & $46.06 \%$ & $33 / 380$ & $8.68 \%$ & $25.68 \%$ & 5 & $\begin{array}{l}3.54- \\
7.07\end{array}$ & 0 & $\begin{array}{l}< \\
0.00001\end{array}$ \\
\hline $\begin{array}{l}\text { Upper } \\
\text { respiratory tract } \\
\text { infection }\end{array}$ & 1 & $20 / 104$ & $19.23 \%$ & $28 / 103$ & $27.18 \%$ & $23.19 \%$ & 0.71 & $\begin{array}{l}0.43- \\
1.17\end{array}$ & - & 0.18 \\
\hline Nasopharyngitis & 2 & $35 / 166$ & $21.08 \%$ & $54 / 228$ & $23.68 \%$ & $22.59 \%$ & 0.73 & $\begin{array}{l}0.51- \\
1.05\end{array}$ & 20 & 0.09 \\
\hline $\begin{array}{l}\text { Increased blood } \\
\text { alkaline } \\
\text { phosphatase }\end{array}$ & 1 & $8 / 62$ & $12.90 \%$ & $33 / 125$ & $26.40 \%$ & $21.93 \%$ & 0.49 & $\begin{array}{l}0.24- \\
0.99\end{array}$ & - & 0.05 \\
\hline AST increased & 2 & $73 / 255$ & $28.63 \%$ & $36 / 255$ & $14.12 \%$ & $21.37 \%$ & 2.03 & $\begin{array}{l}1.41- \\
2.91\end{array}$ & 37 & 0.0001 \\
\hline Pyrexia & 1 & $24 / 104$ & $23.08 \%$ & $14 / 103$ & $13.59 \%$ & $18.36 \%$ & 1.7 & $\begin{array}{l}0.93- \\
3.09\end{array}$ & - & 0.08 \\
\hline $\begin{array}{l}\text { Peripheral } \\
\text { edema }\end{array}$ & 3 & $80 / 317$ & $25.24 \%$ & $46 / 380$ & $12.11 \%$ & $18.08 \%$ & 1.93 & $\begin{array}{l}1.39- \\
2.67\end{array}$ & 0 & $\begin{array}{l}< \\
0.0001\end{array}$ \\
\hline Dysgeusia & 3 & $94 / 317$ & $29.65 \%$ & $25 / 380$ & $6.58 \%$ & $17.07 \%$ & 4.96 & $\begin{array}{l}1.98- \\
12.41\end{array}$ & 63 & 0.0006 \\
\hline $\begin{array}{l}\text { Creatine } \\
\text { phosphokinase } \\
\text { increased }\end{array}$ & 1 & $14 / 104$ & $13.46 \%$ & $21 / 103$ & $20.39 \%$ & $16.91 \%$ & 0.66 & $\begin{array}{l}0.36- \\
1.23\end{array}$ & - & 0.19 \\
\hline Malaise & 1 & $20 / 104$ & $19.23 \%$ & $13 / 103$ & $12.62 \%$ & $15.94 \%$ & 1.52 & $\begin{array}{l}0.80- \\
2.90\end{array}$ & - & 0.2 \\
\hline $\begin{array}{l}\text { Blood bilirubin } \\
\text { increased }\end{array}$ & 3 & 5/317 & $1.58 \%$ & $103 / 380$ & $27.11 \%$ & $15.49 \%$ & 0.07 & $\begin{array}{l}0.03- \\
0.17\end{array}$ & 0 & $\begin{array}{l}< \\
0.00001\end{array}$ \\
\hline $\begin{array}{l}\text { Visual } \\
\text { impairment }\end{array}$ & 2 & $75 / 255$ & $29.41 \%$ & $4 / 255$ & $1.57 \%$ & $15.49 \%$ & 16.55 & $\begin{array}{l}1.42- \\
193.51\end{array}$ & 78 & 0.03 \\
\hline Fatigue & 2 & $35 / 213$ & $16.43 \%$ & $40 / 277$ & $14.44 \%$ & $15.31 \%$ & 1.18 & $\begin{array}{l}0.57- \\
2.41\end{array}$ & 59 & 0.66 \\
\hline $\begin{array}{l}\text { Sinus } \\
\text { bradycardia }\end{array}$ & 2 & $17 / 166$ & $10.24 \%$ & $38 / 228$ & $16.67 \%$ & $13.96 \%$ & 1.51 & $\begin{array}{l}0.16- \\
14.43\end{array}$ & 77 & 0.72 \\
\hline $\begin{array}{l}\text { Decreased } \\
\text { appetite }\end{array}$ & 2 & $44 / 166$ & $26.51 \%$ & $9 / 228$ & $3.95 \%$ & $13.45 \%$ & 7.34 & $\begin{array}{l}3.64- \\
14.77\end{array}$ & 0 & $\begin{array}{l}< \\
0.00001\end{array}$ \\
\hline Rash & 3 & $36 / 317$ & $11.36 \%$ & $55 / 380$ & $14.47 \%$ & $13.06 \%$ & 0.75 & $\begin{array}{l}0.40- \\
1.43\end{array}$ & 52 & 0.39 \\
\hline Bronchitis & 2 & $12 / 166$ & $7.23 \%$ & $36 / 228$ & $15.79 \%$ & $12.18 \%$ & 0.5 & $\begin{array}{l}0.27- \\
0.94\end{array}$ & 0 & 0.03 \\
\hline
\end{tabular}

Abbreviations: AST: aspartate aminotransferase; ALT: alanine aminotransferase; Cl: confidence interval. 


\begin{tabular}{|c|c|c|c|c|c|c|c|c|c|c|}
\hline \multirow[t]{2}{*}{ Adverse events } & \multirow{2}{*}{$\begin{array}{l}\text { Studies } \\
\text { involved }\end{array}$} & \multicolumn{2}{|l|}{ Crizotinib } & \multicolumn{2}{|l|}{ Alectinib } & \multirow{2}{*}{$\begin{array}{l}\text { Total } \\
\text { incidence }\end{array}$} & \multirow{2}{*}{$\begin{array}{l}\text { Risk } \\
\text { ratio }\end{array}$} & \multirow[t]{2}{*}{$95 \% \mathrm{Cl}$} & \multirow[t]{2}{*}{$R(\%)$} & \multirow[t]{2}{*}{$\mathbf{P}$} \\
\hline & & Event/total & $\%$ & Event/total & $\%$ & & & & & \\
\hline Dry skin & 1 & $13 / 104$ & $12.50 \%$ & $12 / 103$ & $11.65 \%$ & $12.08 \%$ & 1.07 & $\begin{array}{l}0.51- \\
2.24\end{array}$ & - & 0.85 \\
\hline Stomatitis & 1 & $12 / 104$ & $11.54 \%$ & $13 / 103$ & $12.62 \%$ & $12.08 \%$ & 0.91 & $\begin{array}{l}0.44- \\
1.91\end{array}$ & - & 0.81 \\
\hline $\begin{array}{l}\text { Neutrophil } \\
\text { count decreased }\end{array}$ & 2 & $39 / 166$ & $23.49 \%$ & $7 / 228$ & $3.07 \%$ & $11.68 \%$ & 7.57 & $\begin{array}{l}3.37- \\
17.03\end{array}$ & 0 & $<.00001$ \\
\hline $\begin{array}{l}\text { Blood creatinine } \\
\text { increased }\end{array}$ & 1 & $11 / 104$ & $10.58 \%$ & $13 / 103$ & $12.62 \%$ & $11.59 \%$ & 0.84 & $\begin{array}{l}0.39- \\
1.78\end{array}$ & - & 0.65 \\
\hline $\begin{array}{l}\text { White blood cell } \\
\text { count decreased }\end{array}$ & 1 & $15 / 62$ & $24.19 \%$ & $6 / 125$ & $4.80 \%$ & $11.23 \%$ & 5.04 & $\begin{array}{l}2.06- \\
12.35\end{array}$ & - & 0.0004 \\
\hline Anemia & 3 & $15 / 317$ & $4.73 \%$ & $58 / 380$ & $15.26 \%$ & $10.47 \%$ & 0.31 & $\begin{array}{l}0.18- \\
0.53\end{array}$ & 0 & $\begin{array}{l}< \\
0.0001\end{array}$ \\
\hline
\end{tabular}

In subgroup analysis of grade 3-5 AEs there was greater ALT increase, AST increase, neutrophil count decrease, electrocardiogram QT prolongation, nausea increase, and vomiting increase associated with the crizotinib group. However, anemia was associated with the alectinib group. Grade 3-5 AEs with an incidence greater than $1 \%$ according to the combination of the crizotinib group and the alectinib group are summarized in Table 4, Table $\mathbf{S} 9$ and Table $\mathbf{S 1 0 .}$ 
Table 4

Grade 3-5 adverse events with an incidence of greater than 10\% according to combination of the two groups.

\begin{tabular}{|c|c|c|c|c|c|c|c|c|c|c|}
\hline \multirow{2}{*}{$\begin{array}{l}\text { Grade } 3-5 \\
\text { adverse events }\end{array}$} & \multirow{2}{*}{$\begin{array}{l}\text { Studies } \\
\text { involved }\end{array}$} & \multicolumn{2}{|l|}{ Crizotinib } & \multicolumn{2}{|l|}{ Alectinib } & \multirow{2}{*}{$\begin{array}{l}\text { Total } \\
\text { incidence }\end{array}$} & \multirow{2}{*}{$\begin{array}{l}\text { Risk } \\
\text { ratio }\end{array}$} & \multirow[t]{2}{*}{$95 \% \mathrm{Cl}$} & \multirow{2}{*}{$\begin{array}{l}R \\
(\%)\end{array}$} & \multirow[t]{2}{*}{$\mathbf{P}$} \\
\hline & & Event/total & $\%$ & Event/total & $\%$ & & & & & \\
\hline ALT increased & 3 & $42 / 317$ & $12.93 \%$ & $10 / 380$ & $2.63 \%$ & $7.32 \%$ & 4.66 & $\begin{array}{l}2.36- \\
9.20\end{array}$ & 0 & $\begin{array}{l}< \\
0.00001\end{array}$ \\
\hline AST increased & 2 & $21 / 255$ & $8.24 \%$ & $9 / 255$ & $3.53 \%$ & $5.88 \%$ & 2.34 & $\begin{array}{l}1.10- \\
5.00\end{array}$ & 0 & 0.03 \\
\hline $\begin{array}{l}\text { Neutrophil count } \\
\text { decreased }\end{array}$ & 3 & $29 / 317$ & $9.15 \%$ & $2 / 380$ & $0.53 \%$ & $4.45 \%$ & 11.41 & $\begin{array}{l}3.64- \\
25.72\end{array}$ & 0 & $\begin{array}{l}<.0001 \\
0.0\end{array}$ \\
\hline $\begin{array}{l}\text { Pulmonary } \\
\text { embolism }\end{array}$ & 1 & $8 / 151$ & $5.30 \%$ & $2 / 152$ & $1.32 \%$ & $3.30 \%$ & 3.41 & $\begin{array}{l}0.84- \\
13.82\end{array}$ & 0 & 0.09 \\
\hline $\begin{array}{l}\text { Interstitial lung } \\
\text { disease }\end{array}$ & 2 & $6 / 166$ & $3.61 \%$ & $6 / 228$ & $2.63 \%$ & $3.05 \%$ & 1.6 & $\begin{array}{l}0.17- \\
15.24\end{array}$ & 66 & 0.68 \\
\hline $\begin{array}{l}\text { Creatine } \\
\text { phosphokinase } \\
\text { increased }\end{array}$ & 1 & $4 / 151$ & $2.65 \%$ & $5 / 152$ & $3.29 \%$ & $2.97 \%$ & 0.79 & $\begin{array}{l}0.22- \\
2.87\end{array}$ & - & 0.72 \\
\hline $\begin{array}{l}\text { Hepatic function } \\
\text { abnormal }\end{array}$ & 1 & $6 / 104$ & $5.77 \%$ & 0/103 & $0.00 \%$ & $2.90 \%$ & 12.88 & $\begin{array}{l}0.73- \\
225.66\end{array}$ & - & 0.08 \\
\hline $\begin{array}{l}\text { Electrocardiogram } \\
\text { QT prolonged }\end{array}$ & 2 & $12 / 255$ & $4.71 \%$ & $2 / 255$ & $0.78 \%$ & $2.75 \%$ & 4.98 & $\begin{array}{l}1.30- \\
19.06\end{array}$ & 0 & 0.02 \\
\hline Pneumonia & 1 & $3 / 151$ & $1.99 \%$ & $4 / 152$ & $2.63 \%$ & $2.31 \%$ & 0.75 & $\begin{array}{l}0.17- \\
3.32\end{array}$ & - & 0.71 \\
\hline Neutropenia & 1 & $6 / 151$ & $3.97 \%$ & $0 / 152$ & $0.00 \%$ & $1.98 \%$ & 13.09 & $\begin{array}{l}0.74- \\
230.25\end{array}$ & - & 0.08 \\
\hline Anemia & 2 & $1 / 255$ & $0.39 \%$ & $9 / 255$ & $3.53 \%$ & $1.96 \%$ & 0.16 & $\begin{array}{l}0.03- \\
0.88\end{array}$ & 0 & 0.04 \\
\hline $\begin{array}{l}\text { Maculopapular } \\
\text { rash }\end{array}$ & 1 & $1 / 104$ & $0.96 \%$ & $3 / 103$ & $2.91 \%$ & $1.93 \%$ & 0.33 & $\begin{array}{l}0.03- \\
3.12\end{array}$ & - & 0.33 \\
\hline Hyponatremia & 2 & $6 / 213$ & $2.82 \%$ & $3 / 277$ & $1.08 \%$ & $1.84 \%$ & 2.8 & $\begin{array}{l}0.21- \\
36.84\end{array}$ & 60 & 0.43 \\
\hline $\begin{array}{l}\text { Urinary tract } \\
\text { infection }\end{array}$ & 1 & $1 / 151$ & $0.66 \%$ & $4 / 152$ & $2.63 \%$ & $1.65 \%$ & 0.25 & $\begin{array}{l}0.03- \\
2.23\end{array}$ & - & 0.21 \\
\hline $\begin{array}{l}\text { Increased bilirubin } \\
\text { conjugated }\end{array}$ & 1 & $1 / 62$ & $1.61 \%$ & $2 / 125$ & $1.60 \%$ & $1.60 \%$ & 1.01 & $\begin{array}{l}0.09- \\
10.90\end{array}$ & - & 0.99 \\
\hline Nausea & 3 & $9 / 317$ & $2.84 \%$ & $2 / 380$ & $0.53 \%$ & $1.58 \%$ & 4.71 & $\begin{array}{l}1.14- \\
19.44\end{array}$ & 0 & 0.03 \\
\hline $\begin{array}{l}\text { Photosensitivity } \\
\text { reaction }\end{array}$ & 1 & $3 / 151$ & $1.99 \%$ & $1 / 152$ & $0.66 \%$ & $1.32 \%$ & 3.02 & $\begin{array}{l}0.32- \\
28.71\end{array}$ & - & 0.34 \\
\hline Pneumonitis & 1 & 4/151 & $2.65 \%$ & $0 / 152$ & $0.00 \%$ & $1.32 \%$ & 9.06 & $\begin{array}{l}0.49- \\
166.82\end{array}$ & - & 0.14 \\
\hline Pleural effusion & 1 & $2 / 151$ & $1.32 \%$ & $2 / 152$ & $1.32 \%$ & $1.32 \%$ & 1.01 & $\begin{array}{l}0.14- \\
7.05\end{array}$ & - & 0.99 \\
\hline Acute kidney injury & 1 & 0/151 & $0.00 \%$ & $4 / 152$ & $2.63 \%$ & $1.32 \%$ & 0.11 & $\begin{array}{l}0.01- \\
2.06\end{array}$ & - & 0.14 \\
\hline $\begin{array}{l}\text { Decreased } \\
\text { appetite }\end{array}$ & 2 & $4 / 166$ & $2.41 \%$ & $1 / 228$ & $0.44 \%$ & $1.27 \%$ & 4.23 & $\begin{array}{l}0.80- \\
22.47\end{array}$ & 41 & 0.09 \\
\hline $\begin{array}{l}\text { White blood cell } \\
\text { count decreased }\end{array}$ & 2 & $5 / 166$ & $3.01 \%$ & $0 / 228$ & $0.00 \%$ & $1.27 \%$ & 7.75 & $\begin{array}{l}0.87- \\
68.81\end{array}$ & 0 & 0.07 \\
\hline
\end{tabular}

Abbreviations: AST: aspartate aminotransferase; ALT: alanine aminotransferase; Cl: confidence interval. 


\begin{tabular}{|c|c|c|c|c|c|c|c|c|c|c|}
\hline \multirow{2}{*}{$\begin{array}{l}\text { Grade } 3-5 \\
\text { adverse events }\end{array}$} & \multirow{2}{*}{$\begin{array}{l}\text { Studies } \\
\text { involved }\end{array}$} & \multicolumn{2}{|l|}{ Crizotinib } & \multicolumn{2}{|l|}{ Alectinib } & \multirow{2}{*}{$\begin{array}{l}\text { Total } \\
\text { incidence }\end{array}$} & \multirow{2}{*}{$\begin{array}{l}\text { Risk } \\
\text { ratio }\end{array}$} & \multirow[t]{2}{*}{$95 \% \mathrm{Cl}$} & \multirow{2}{*}{$\begin{array}{l}R \\
(\%)\end{array}$} & \multirow[t]{2}{*}{$\mathbf{P}$} \\
\hline & & Event/total & $\%$ & Event/total & $\%$ & & & & & \\
\hline Weight increased & 2 & $1 / 213$ & $0.47 \%$ & $5 / 277$ & $1.81 \%$ & $1.22 \%$ & 0.44 & $\begin{array}{l}0.07- \\
2.65\end{array}$ & 0 & 0.37 \\
\hline Vomiting & 3 & $7 / 317$ & $2.21 \%$ & $0 / 380$ & $0.00 \%$ & $1.00 \%$ & 10.64 & $\begin{array}{l}1.28- \\
88.57\end{array}$ & 0 & 0.03 \\
\hline Rash & 3 & $3 / 317$ & $0.95 \%$ & $4 / 380$ & $1.05 \%$ & $1.00 \%$ & 1 & $\begin{array}{l}0.29- \\
3.44\end{array}$ & 41 & 1 \\
\hline
\end{tabular}

\section{Publication bias}

The funnel plots for publication bias according to the summary of survival (Figure S6A) and safety (Figure S6B) demonstrated marked evidence of symmetry, indicating an acceptable publication bias. The combined effect size (summary of survival, $Z$ value of $14.56, P<$ 0.00001 ; summary of safety, $\mathrm{Z}$ value of $4.99, \mathrm{P}<0.00001$ ) indicated that the fail-safe $\mathrm{N}$ value was relevant.

\section{Discussion}

ALK-positive lung cancer accounts for 3\%-7\% of all lung cancer cases and, in the past, was one of the genetic markers that indicated poor prognosis ${ }^{[2,19]}$. Recently, survival of patients with ALK-positive NSCLC has been greatly improved following the discovery and use of ALK inhibitors ${ }^{[20,21]}$. As the representative 1 st and 2 nd generation ALK inhibitors, crizotinib and alectinib have been widely used in clinical practice, and their efficacy and safety have been determined ${ }^{[22,23]}$. However, whether alectinib exhibits superior antitumor efficacy than crizotinib for the treatment of ALK-positive NSCLC, especially as a first-line treatment, remains controversial ${ }^{[9,10]}$. This is the first meta-analysis performed that focuses on the comparison of crizotinib to alectinib in ALK-positive NSCLC patients based on three high quality RCTs (the ALEX, ALESIA and J-ALEX clinical trials). In summary, the alectinib therapy had better efficacy compared with the crizotinib therapy in OS, PFS, CNS-PFS, DOR, ORR, PR and grade 3-5 AEs. Additionally, the survival advantages of alectinib compared with crizotinib increased with the prolongation of survival time. Similar DCR, CR and total AEs were found in the two groups.

Better survival, especially control of the CNS metastases, is the primary benefit of alectinib treatment. The advantages of alectinib are significant in both first-line and second-line treatment of ALK-positive NSCLC. Camidge et al. ${ }^{[23]}$ analyzed the updated data of the ALEX study and found that the alectinib therapy could greatly prolong the PFS of patients with ALK-positive NSCLC compared with the crizotinib therapy (34.8 months vs. 10.9 months, HR: $0.43,95 \% \mathrm{Cl}$ : 0.32-0.58). Similar results were confirmed in the ALESIA study [14]. Nishio et al. ${ }^{[13]}$ analyzed the CNS efficacy in the J-ALEX trial and found that alectinib could delay brain metastasis and averted the progression of brain metastases. The Gadgeel et al's study ${ }^{[22]}$ based on the ALEX trial reported superior CNS activity of the alectinib group therapy for ALK-positive NSCLC regardless of CNS disease or radiotherapy at baseline. Five reasons may explain the benefits observed with the alectinib therapy. First, as a highly selective ALK inhibitor, alectinib combines a significantly different ALK tyrosine kinase domain from crizotinib, which can overcome the secondary mutation of kinase domain caused by crizotinib [25]. Second, alectinib can inhibit more ALK-mutations (such as EML4-ALK, G1269A, C1156Y, F1174L, 1151Tin and L1152R), which were identified in lung cancer tissues of crizotinib-resistant patients ${ }^{[6]}$. Third, alectinib has a stronger affinity for the ALK tyrosine kinase domain, which can increase the depth of response and prolong the DOR (HR: 0.31, [0.23-0.42], $p<0.00001$ ), resulting in a longer PFS [26]. Fourth, alectinib (which is not a substrate of P-glycoprotein) can penetrate into the CNS more effectively than crizotinib and delay brain metastasis ${ }^{[27]}$. Fifth, a higher frequency of AEs during crizotinib therapy resulted in shorter treatment durations ${ }^{[11,12,14]}$. Subgroup analysis of PFS suggested that being an active smoker, having an ECOG-PS of 2, having postoperative recurrence, and the ALK testing method being RT-PCR might be unfavorable factors for alectinib treatment. However, baseline CNS metastases and previous brain radiation might be favorable factors for alectinib treatment. Additionally, the survival advantages of alectinib compared with crizotinib increased with the prolongation of survival. Ito et al. and Watanabe et al. reported that sequential therapy with crizotinib and alectinib after crizotinib failure could provide a better survial benefit than did therapy with alectinib or crizotinib alone in patients with ALKpositive NSCLC ${ }^{[28,29]}$. Alectinib was also approved by FDA for the treatment of metastatic, ALK + NSCLC following crizotinib ${ }^{[21]}$. In 
summary, we believe that alectinib is a better choice for ALK-positive NSCLC first line treatment or second line treatment, especially for patients with baseline CNS metastases and/or previous brain radiation.

Relatively good safety is another advantage of treatment with alectinib, although alectinib does have a longer treatment duration. Thirteen AEs with an incidence of $>20 \%$ were reported in the crizotinib group (nausea, diarrhea, constipation, vomiting, increased ALT, dysgeusia, visual impairment, increased AST, decreased appetite, peripheral edema, decreased neutrophil count, pyrexia and nasopharyngitis) compared with eight AEs in the alectinib group (constipation, upper respiratory tract infection, increased blood bilirubin, increased bilirubin conjugated, increased blood alkaline phosphatase, nasopharyngitis, increased ALT and increased creatine phosphokinase). Additionally, fifteen grade 3-5 AEs were reported with an incidence greater than $2 \%$ in the crizotinib group (increased ALT, decreased neutrophil count, increased AST, abnormal hepatic function, pulmonary embolism, prolonged electrocardiogram QT, neutropenia, interstitial lung disease, decreased white blood cell count, nausea, hyponatremia, increased creatine phosphokinase, pneumonitis, decreased appetite and vomiting) compared with nine in the alectinib group (anemia, increased AST, increased creatine phosphokinase, maculopapular rash, pneumonia, interstitial lung disease, increased ALT, urinary tract infection and acute kidney injury). The frequency of AEs were similar to previous reports Seto et al. ${ }^{\left[{ }^{[8}\right.}$ in the phase $1-2$ studies. Hida et al. ${ }^{[12]}$ reported that crizotinib treatment achieved more discontinuations and dose interruptions caused by AEs, which might also decrease the efficacy of crizotinib. Hou et al. ${ }^{[30]}$ evaluated the safety of all approved ALK inhibitors and suggested that alectinib was the safest ALK inhibitor. In the subgroup analysis, more gastrointestinal disorders, nervous system disorders, eye disorders and metabolism disorders were found in the crizotinib group. However, the alectinib treatment seemed to cause more AEs in the urinary system, musculoskeletal and connective tissue, and the respiratory system. In summary, although alectinib is safer than crizotinib for patients with ALK-positive NSCLC, its high frequency of grade 3-5 AEs should be considered during treatment.

The current meta-analysis had several limitations. First, we only included articles written in English, which introduces a language bias. Second, only 3 RCTs were included, which decreases the clinical value of the combined data. Third, like most other meta-analysis, all data in our analysis were extracted from previously published articles, which increases the heterogeneity of data in the merger analysis. Fourth, 532/697 patients came from Asia, which decreases the clinical value in other countries. Fifth, significant heterogeneity was found in the analysis of OSR, CNS-PFSR-6 $\mathrm{m}$ and DCR, which decreased the quality of these results. Sixth, individual patient data meta-analysis and treatment sequence of crizotinib and alectinib were not conducted due to lack of data, which might decrease the clinical value of results. Seventh, the median follow up time was different, which might increase data heterogeneity between the included studies.

\section{Conclusion}

Alectinib exhibited better OS, PFS, CNS-PFS and safety compared with crizotinib; thus, alectinib appears to be superior to crizotinib for the treatment of ALK-positive NSCLC. The survival advantages of alectinib increased with the prolongation of survival time. Baseline CNS metastases and previous brain radiation might be favorable factors for alectinib treatment. Although alectinib is safer than crizotinib, its high frequency of grade $3-5 \mathrm{AEs}(37.37 \%)$ should be considered during treatment. Additionally, the existing shortcomings of this meta-analysis require further extensive and high-quality trials to resolve and confirm our conclusions.

\section{Abbreviations}

ALK: anaplastic lymphoma kinase; NSCLC: non-small cell lung cancer; OS: overall survival; PFS: progression-free survival; CNS: central nervous system; AEs: adverse effects; HR: hazard ratio; RR: Risk ratio; Cl: confidence interval; ORR: objective response rate; PR: partial response; DCR: disease control rate; CR: complete response; AST: aspartate aminotransferase; ALT: alanine aminotransferase; PRISMA: Preferred Reporting Items for Systematic Review and Meta-Analysis guidelines; RCT: randomized controlled trial; M/F: male/female; Bid: twice a day; SD: stable diease; DOR: duration of response; OSR: overall survival rate; PFSR: progression-free survival rate; CNSPFSR: central nervous system progression-free survival rate; ECOG-PS: Eastern Cooperative Oncology Group-performance status; GRADE: Grading of Recommendations Assessment, Development and Evaluation; RT-PCR: reverse transcription-polymerase chain reaction; IRC: independent review committee; IHC: immunohistochemistry; FISH: fluorescence in situ hybridization; MRI: magnetic resonance imaging; RECIST: Response Evaluation Criteria In Solid Tumors; RT-PCR: Reverse Transcription-Polymerase Chain Reaction.

\section{Declarations}


Funding: This study was supported by National Natural Science Foundation of China (NSFC), number of grants (81560345), Natural Science Foundation of Jiangxi Province (Grant number: 20181BBG78023). The funding had no role in the design and conduct of the study; collection, management, analysis, and interpretation of the data; preparation, review, or approval of the manuscript; and decision to submit the manuscript for publication.

Conflicts of interest/Competing interests: The authors certify that there is no confict of interest regarding this manuscript.

Ethics approval: This article does not contain any studies with human participants or animals performed by any of the authors.

Informed consent: For this type of study formal consent is not required.

Availability of data and material: The data sets used and/or analysed during the current study are available from the corresponding author on reasonable request.

Authors' contributions: Qinghua Zeng had full access to all of the data in the manuscript and takes responsibility for the integrity of the data and the accuracy of the data analysis.

Concept and design: All authors.

Acquisition, analysis, or interpretation of data: All authors.

Drafting of the manuscript: Qinghua Zeng and Lin Zeng.

Critical revision of the manuscript for important intellectual content: Qinghua Zeng, Xiquan Zhang, Shan He, Zhiyong Zhou, Luping Xia and Wenxiong Zhang.

Statistical analysis: Qinghua Zeng, Lin Zeng and Wenxiong Zhang.

Supervision: Qinghua Zeng and Wenxiong Zhang.

Acknowledgements: The authors thank professor Jichun Liu, MD (Department of Cardio-Thoracic Surgery, The second affiliated hospital of Nanchang University) for his advice and professor Xiaoshu Cheng, MD, PhD (Department of Cardiology, The second affiliated hospital of Nanchang University) for his data collection.

\section{References}

1. Siegel RL, Miller KD, Jemal A. Cancer statistics, 2020. CA Cancer J Clin. 2020;70(1):7-30.

2. Dearden S, Stevens J, Wu YL, Blowers D. Mutation incidence and coincidence in non small-cell lung cancer: meta-analyses by ethnicity and histology (mutMap). Ann Oncol. 2013;24:2371-2376.

3. Solomon BJ, Mok T, Kim DW, Wu YL, Nakagawa K, Mekhail T, et al. First-line crizotinib versus chemotherapy in ALK-positive lung cancer. N Engl J Med. 2014; 371:2167-2177.

4. Shaw AT, Kim DW, Nakagawa K, Seto T, Crinó L, Ahn MJ, et al. Crizotinib versus chemotherapy in advanced ALK-positive lung cancer. N Engl J Med. 2013; 368:2385-2394.

5. Gridelli C, Peters S, Sgambato A, Casaluce F, Adjei AA, Ciardiello F. ALK inhibitors in the treatment of advanced NSCLC. Cancer Treat Rev. 2014;40:300-306.

6. Kodama T, Tsukaguchi T,Yoshida M, Kondoh O, Sakamoto H. Selective ALK inhibitor alectinib with potent antitumor activity in models of crizotinib resistance. Cancer Lett. 2014; 351:215-21

7. Iwama E, Goto Y, Murakami H, Tsumura S, Sakashita H, Mori Y, et al. Survival Analysis for Patients with ALK RearrangementPositive Non-Small Cell Lung Cancer and a Poor Performance Status Treated with Alectinib: Updated Results of Lung Oncology Group in Kyushu 1401. Oncologist. 2019. pii: theoncologist.2019-0728.

8. Seto T, Kiura K, Nishio M, Nakagawa K, Maemondo M, Inoue A, et al. CH5424802 (R05424802) for patients with ALK-rearranged advanced non-small-cell lung cancer (AF-001JP study): a single-arm, open-label, phase 1-2 study. Lancet Oncol. 2013;14:590-598.

9. Ettinger DS, Wood DE, Aggarwal C, Aisner DL, Akerley W, Bauman JR, et al. NCCN Guidelines Insights: Non-Small Cell Lung Cancer, Version 1.2020. J Natl Compr Canc Netw. 2019;17(12):1464-1472. 
10. Planchard D, Popat S, Kerr K, Novello S, Smit EF, Faivre-Finn C, et al. Metastatic non-small cell lung cancer: ESMO Clinical Practice Guidelines for diagnosis, treatment and follow-up. Ann Oncol. 2018;29(Suppl 4):iv192-iv237.

11. Peters S, Camidge DR, Shaw AT, Gadgeel S, Ahn JS, Kim DW, et al. Alectinib versus crizotinib in untreated ALK-positive non-smallcell lung cancer. N Engl J Med. 2017; 377:829-838.

12. Hida T, Nokihara H, Kondo M, Kim YH, Azuma K, Seto T, et al. Alectinib versus crizotinib in patients with ALK-positive non-small-cell lung cancer (J-ALEX): an open-label, randomised phase 3 trial. Lancet. 2017;390(10089):29-39.

13. Nishio M, Nakagawa K, Mitsudomi T, Yamamoto N, Tanaka T, Kuriki H, et al. Analysis of central nervous system efficacy in the JALEX study of alectinib versus crizotinib in ALK-positive non-small-cell lung cancer. Lung Cancer. 2018;121:37-40.

14. Zhou C, Kim SW, Reungwetwattana T, Zhou J, Zhang Y, He J, et al. Alectinib versus crizotinib in untreated Asian patients with anaplastic lymphoma kinase-positive non-small-cell lung cancer (ALESIA): a randomised phase 3 study. Lancet Respir Med. 2019;7(5):437-446.

15. Bedas A, Peled N, Maimon Rabinovich N, Mishaeli M, Shochat T, Zer A, et al. Efficacy and Safety of ALK Tyrosine Kinase Inhibitors in Elderly Patients with Advanced ALK-Positive Non-Small Cell Lung Cancer: Findings from the Real-Life Cohort. Oncol Res Treat. 2019;42(5):275-282.

16. Jadad AR, Moore RA, Carroll D, Jenkinson C, Reynolds DJ, Gavaghan DJ, et al. Assessing the quality of reports of randomized clinical trials: is blinding necessary? Control Clin Trials. 1996; 17(1): 1-12.

17. Guyatt GH, Oxman AD, Schünemann HJ, Tugwell P, Knottnerus A. GRADE guidelines: a new series of articles in the Journal of Clinical Epidemiology. J Clin Epidemiol. 2011; 64(4): 380-382.

18. Higgins JP, Altman DG, Gøtzsche PC, Jüni P, Moher D, Oxman AD, et al. The Cochrane Collaboration's tool for assessing risk of bias in randomised trials. BMJ. 2011;343:d5928.

19. He Y, Gong R, Sun LY, Zhang ZC, Liu XY, Shao Q, et al. The Percentage of Anaplastic Lymphoma Kinase-Positive Tumor Cells Has Clinical Implications for Patients with Non-Small Cell Lung Cancer. Genet Test Mol Biomarkers. 2019;23(8):589-597.

20. Malik SM, Maher VE, Bijwaard KE, Becker RL, Zhang L, Tang SW, et al. U.S. Food and Drug Administration approval: crizotinib for treatment of advanced or metastatic non-small cell lung cancer that is anaplastic lymphoma kinase positive. Clin Cancer Res. 2014;20(8):2029-2034.

21. Larkins E, Blumenthal GM, Chen H, He K, Agarwal R, Gieser G, et al. FDA Approval: Alectinib for the Treatment of Metastatic, ALKPositive Non-Small Cell Lung Cancer Following Crizotinib. Clin Cancer Res. 2016;22(21):5171-5176.

22. Gadgeel S, Peters S, Mok T, Shaw AT, Kim DW, Ou SI, et al. Alectinib versus crizotinib in treatment-naive anaplastic lymphoma kinase-positive (ALK+) non-small-cell lung cancer: CNS efficacy results from the ALEX study. Ann Oncol. 2018;29(11):2214-2222.

23. Camidge DR, Dziadziuszko R, Peters S, Mok T, Noe J, Nowicka M, et al. Updated Efficacy and Safety Data and Impact of the EML4ALK Fusion Variant on the Efficacy of Alectinib in Untreated ALK-Positive Advanced Non-Small Cell Lung Cancer in the Global Phase III ALEX Study. J Thorac Oncol. 2019;14(7):1233-1243.

24. Nakagawa K, Hida T, Nokihara H, Morise M, Azuma K, Kim YH, et al. Final progression-free survival results from the J-ALEX study of alectinib versus crizotinib in ALK-positive non-small-cell lung cancer. Lung Cancer. 2020;139:195-199.

25. Karachaliou N, Fernandez Bruno M, Bracht JWP, Rosell R. Profile of alectinib for the treatment of ALK-positive non-small cell lung cancer (NSCLC): patient selection and perspectives. Onco Targets Ther. 2019;12:4567-4575.

26. Novello S, Mazières J, Oh IJ, de Castro J, Migliorino MR, Helland $\AA$, et al. Alectinib versus chemotherapy in crizotinib-pretreated anaplastic lymphoma kinase (ALK)-positive non-small-cell lung cancer: results from the phase III ALUR study. Ann Oncol. 2018;29(6):1409-1416.

27. Kodama T, Hasegawa M, Takanashi K, Sakurai Y, Kondoh O, Sakamoto H. Antitumor activity of the selective ALK inhibitor alectinib in models of intracranial metastases. Cancer Chemother Pharmacol 2014;74:1023-1028.

28. Ito K, Hataji O, Kobayashi H, Fujiwara A, Yoshida M, D'Alessandro-Gabazza CN, et al. Sequential Therapy with Crizotinib and Alectinib in ALK-Rearranged Non-Small Cell Lung Cancer-A Multicenter Retrospective Study. Thorac Oncol. 2017;12(2):390-396.

29. Watanabe S, Hayashi H, Okamoto K, Fujiwara K, Hasegawa Y, Kaneda H, et al. Progression-Free and Overall Survival of Patients With ALK Rearrangement-Positive Non-Small Cell Lung Cancer Treated Sequentially With Crizotinib and Alectinib. Clin Lung Cancer. 2016;17(6):528-534.

30. Hou H, Sun D, Liu K, Jiang M, Liu D, Zhu J, et al. The safety and serious adverse events of approved ALK inhibitors in malignancies: a meta-analysis. Cancer Manag Res. 2019;11:4109-4118.

Page $15 / 21$ 
Figures

PRISMA 2009 Flow Diagram

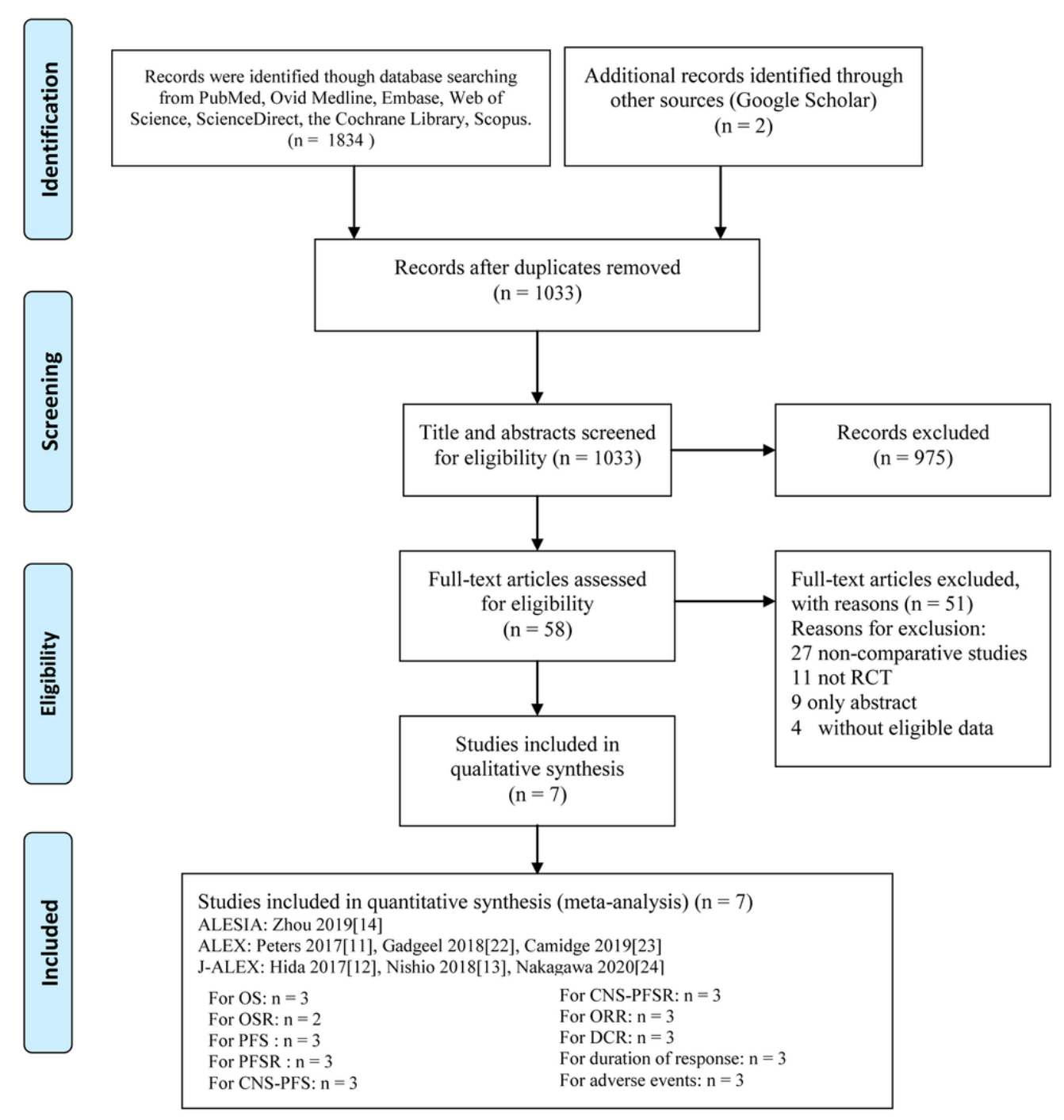

\section{Figure 1}

Flow chart of study selection. 


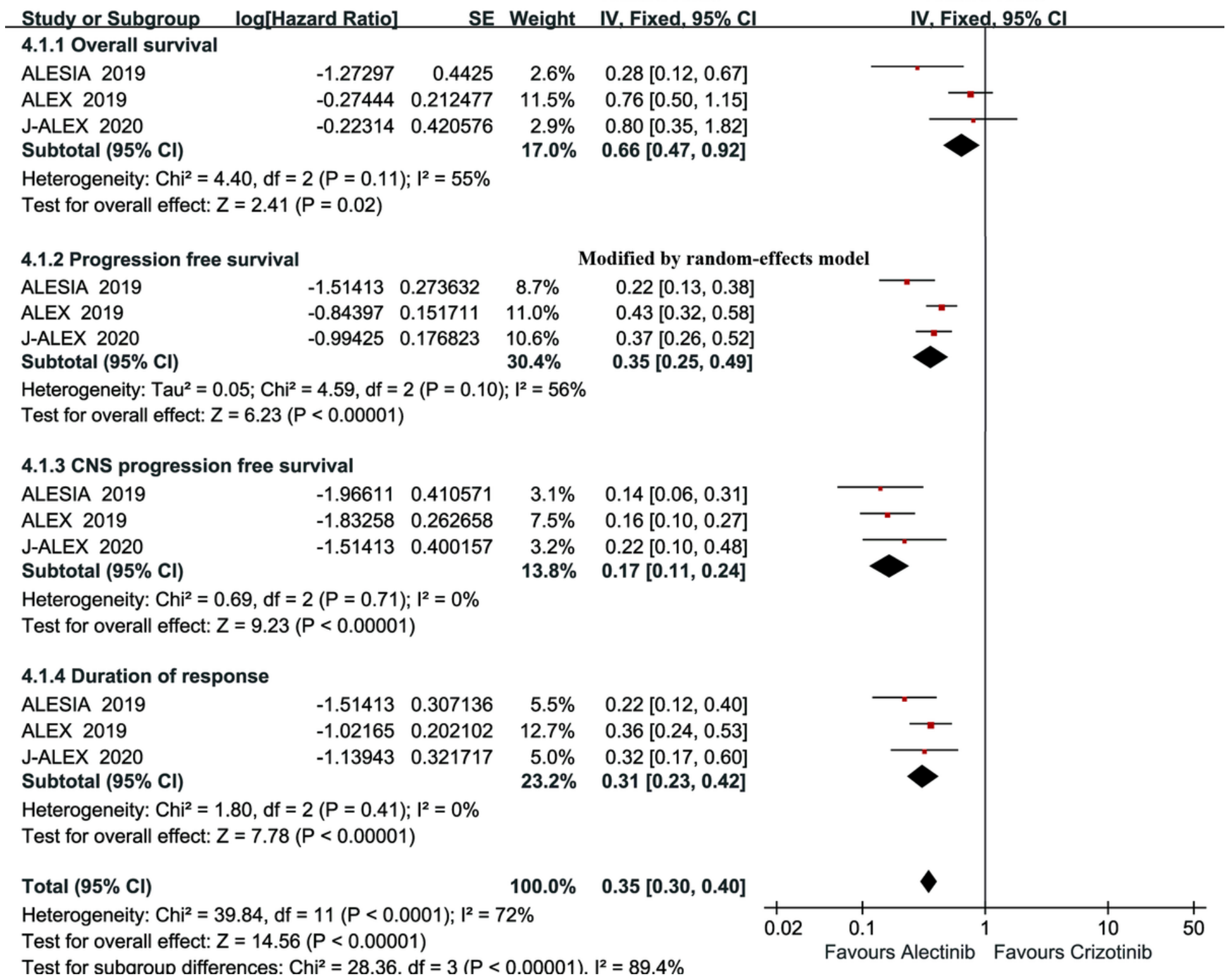

Figure 2

Forest plots of OS, PFS, CNS-PFS and DOR associated with crizotinib versus alectinib. 

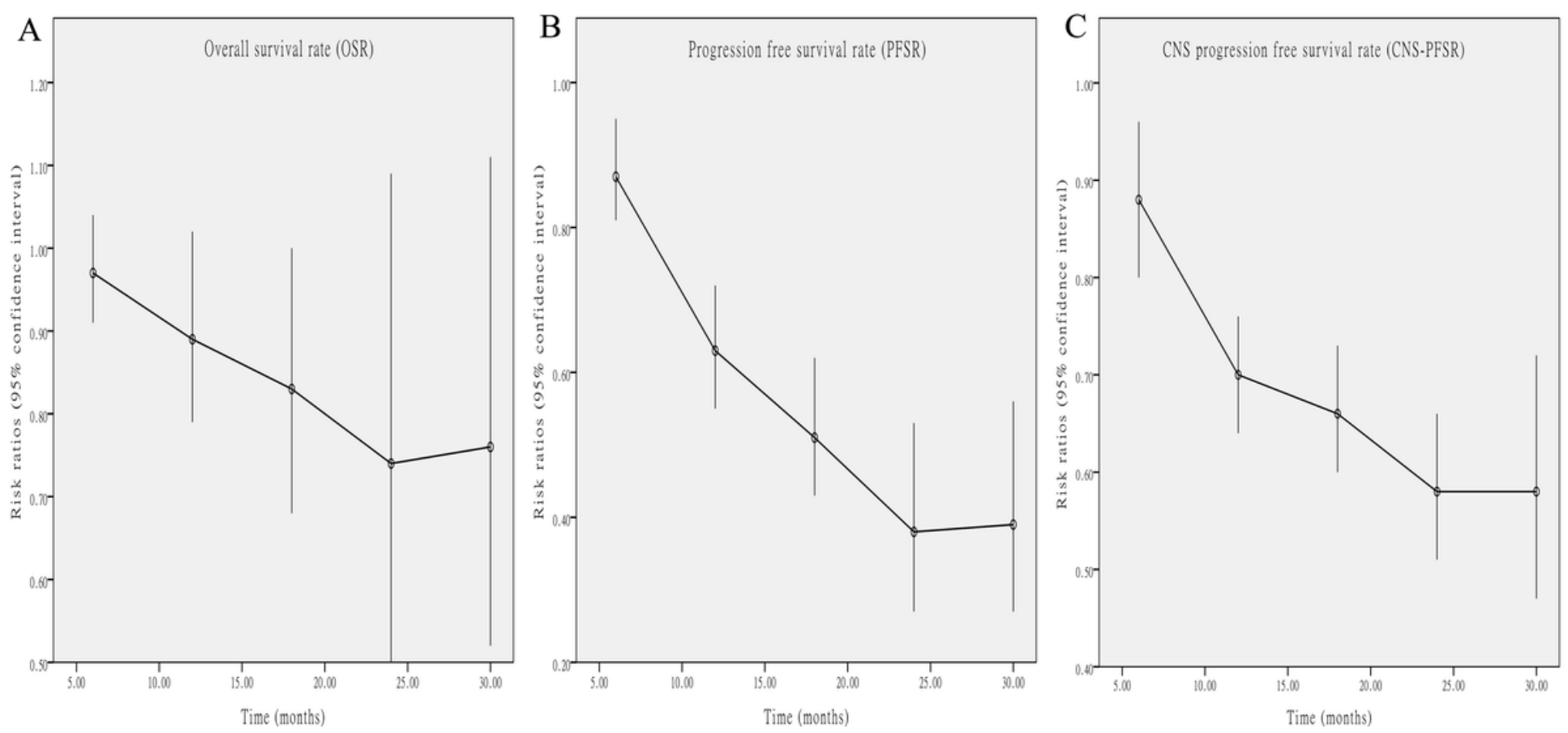

\section{Figure 3}

Line charts of OSR (6-30 months, A), BFSR (6-30 months, B) and CNS-PFSR (6-30 months, C) associated with crizotinib versus alectinib according to survival time. 


\begin{tabular}{|c|c|c|c|c|c|c|c|c|c|}
\hline \multirow{2}{*}{ Subgroups } & \multirow{2}{*}{$\begin{array}{l}\text { Included } \\
\text { studies }\end{array}$} & \multirow{2}{*}{ Total } & \multicolumn{2}{|c|}{ Crizotinib } & \multicolumn{2}{|c|}{ Alectinib } & & & \multirow{2}{*}{ HR $(95 \% \mathrm{CI})$} \\
\hline & & & Events & $\mathrm{n}$ & Events & $\mathrm{n}$ & & & \\
\hline All patients & 3 & 697 & 211 & 317 & 123 & 380 & - & & $0.35(0.25,0.49)$ \\
\hline \multicolumn{10}{|l|}{ Age } \\
\hline$<65$ years & 3 & 584 & 173 & 264 & 102 & 320 & & & $0.37(0.29,0.47)$ \\
\hline$>65$ years & 3 & 113 & 38 & 53 & 21 & 60 & & & $0.35(0.20,0.62)$ \\
\hline \multicolumn{10}{|l|}{ Sex } \\
\hline Female & 3 & 385 & 114 & 178 & 65 & 207 & & & $0.35(0.26,0.49)$ \\
\hline Male & 3 & 312 & 97 & 139 & 58 & 173 & & & $0.32(0.16,0.64)$ \\
\hline \multicolumn{10}{|l|}{ Smoking status } \\
\hline Active smoker & 3 & 114 & 32 & 51 & 15 & 63 & & & $0.42(0.08,2.24)$ \\
\hline Former smoker & 2 & 147 & 47 & 62 & 28 & 85 & & & $0.27(0.10,0.69)$ \\
\hline Non-smoker & 3 & 436 & 132 & 184 & 80 & 232 & & & $0.40(0.30,0.53)$ \\
\hline \multicolumn{10}{|l|}{ ECOG PS } \\
\hline 0 & 3 & 326 & 98 & 164 & 46 & 162 & & & $0.36(0.25,0.52)$ \\
\hline 1 & 2 & 342 & 102 & 140 & 67 & 202 & & & $0.30(0.15,0.62)$ \\
\hline 2 & 3 & 29 & 11 & 13 & 10 & 16 & & & $0.79(0.29,2.16)$ \\
\hline \multicolumn{10}{|l|}{ CNS metastases at baseline } \\
\hline Yes & 3 & 232 & 84 & 110 & 42 & 122 & - & & $0.18(0.07,0.48)$ \\
\hline No & 3 & 465 & 127 & 207 & 81 & 258 & & & $0.42(0.31,0.55)$ \\
\hline \multicolumn{10}{|l|}{ Previous brain radiation } \\
\hline Yes & 2 & 60 & 23 & 26 & 14 & 34 & & & $0.23(0.11,0.49)$ \\
\hline No & 2 & 430 & 130 & 187 & 84 & 243 & & & $0.36(0.19,0.66)$ \\
\hline \multicolumn{10}{|l|}{ Race category } \\
\hline Asian & 3 & 532 & 146 & 235 & 83 & 297 & & & $0.34(0.26,0.44)$ \\
\hline Non-asian & 1 & 165 & 65 & 82 & 40 & 83 & & & $0.44(0.30,0.65)$ \\
\hline \multicolumn{10}{|l|}{ Treatment line } \\
\hline First line & 3 & 623 & 188 & 280 & 113 & 343 & & & $0.36(0.28,0.46)$ \\
\hline Second line & 1 & 74 & 23 & 37 & 10 & 37 & & & $0.39(0.18,0.84)$ \\
\hline \multicolumn{10}{|l|}{ Disease stage } \\
\hline Stage IIIB or IV & 3 & 647 & 198 & 291 & 117 & 356 & i & & $0.36(0.28,0.45)$ \\
\hline Postoperative recurrence & 1 & 50 & 13 & 26 & 6 & 24 & & & $0.49(0.18,1.32)$ \\
\hline \multicolumn{10}{|l|}{ ALK testing method } \\
\hline Single confirmation (IHC or RT-PCR) & 3 & 507 & 159 & 223 & 102 & 284 & & & $0.38(0.30,0.50)$ \\
\hline \multirow[t]{2}{*}{ Double confirmation (IHC and FISH) } & 1 & 190 & 52 & 94 & 21 & 96 & & & $0.30(0.18,0.50)$ \\
\hline & & & & & & & $\begin{array}{ll}.01 & 0.1 \\
& 0.01 \\
& \text { Favours Alectinib }\end{array}$ & $\begin{array}{c}10 \\
\text { Favours Crizotinib }\end{array}$ & \\
\hline
\end{tabular}

Figure 4

Forest plots of PFS in patient subgroups. 


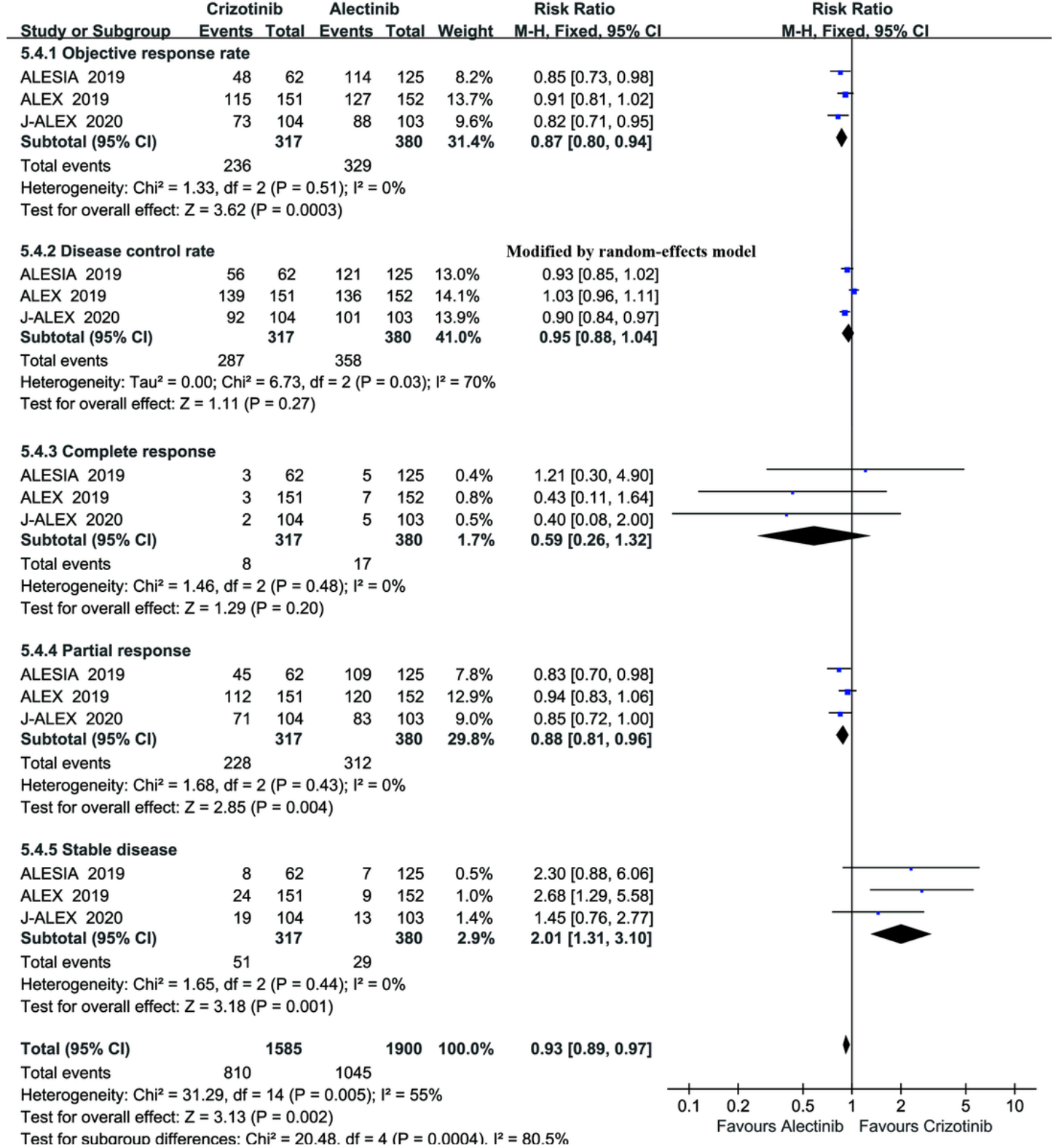

\section{Figure 5}

Forest plots of drug responses (ORR, DCR, CR, PR and SD) associated with crizotinib versus alectinib.

\section{Supplementary Files}

This is a list of supplementary files associated with this preprint. Click to download.

- Figures1.tif

- Figures2.tif

- Figures3.tif

- Figures4.tif

- Figures5.tif

- Figures6.tif

- TableS1.doc

- Tables2.docx

- TableS3.doc 
- Tables4.doc

- TableS5.doc

- Tables6.doc

- Tables7.doc

- Tables8.doc

- Tables9.doc

- TableS10.doc 